\title{
Micromagnetic Modelling of the Dynamics of Exchange Springs in Multi-Layer Systems
}

\author{
M. Franchin ${ }^{1,2}$, J. P. Zimmermann ${ }^{1}$, T. Fischbacher ${ }^{1}$, G. Bordignon ${ }^{1,2}$, P. A. J. de Groot ${ }^{2}$, and H. Fangohr ${ }^{1}$ \\ ${ }^{1}$ School of Engineering Sciences, University of Southampton, Southampton SO17 1BJ, U.K. \\ ${ }^{2}$ School of Physics and Astronomy, University of Southampton, Southampton SO17 1BJ, U.K.
}

Exchange springs are formed in multilayers of alternating hard and soft ferromagnetic materials which are exchange coupled at their interfaces. These systems are rich of interesting physical properties, which can be tuned by selecting suitable geometries and compositions. In this paper, we present a computational study of the dynamics of a tri-layer $\mathrm{DyFe}_{2} / \mathrm{YFe}_{2} / \mathrm{DyFe}_{2}$ exchange spring system near the bending field (the field required to twist the magnetization of the soft $\mathrm{YFe}_{2}$ layer out of the aligned state). The dynamical reaction of the system to small variations of the applied field is studied and its oscillatory nature is analyzed numerically. The behaviors of the decay times, the frequencies, and amplitudes reveal enhanced responses of the system near the bending field.

Index Terms-Dysprosium alloys, exchange springs, finite-element method, magnetic layered films, magnetic multilayers, micromagnetism, Nmag, numerical analysis, yttrium alloys.

\section{INTRODUCTION}

$\mathbf{E}$ XCHANGE spring systems are composites of magnetically hard and soft materials that are exchange coupled across their interfaces. They are promising candidates for many technological applications. They could be used to obtain high densities in storage media, while keeping acceptable writability and thermal stability [1]. They could also be used as highperformance permanent magnets [2] or as giant magneto resistance (GMR) sensors. An important feature of these systems appears when a suitable external field is applied: the magnetization of the soft material responds in a position dependent way, being bounded to the magnetization of the hard material near the interfaces and twisting towards the applied field in the bulk. This resembles a mechanical torsion spring, thus comes the name "exchange spring." The torsion is exhibited only when the applied field exceeds a particular value called the bending field. For lower values the fully aligned state is preferred, because it turns out to minimize the energy, as shown by simple theoretical models [3]. The slope of the magnetization (with respect to varying the applied field) is large close to the bending field. This may be of technological relevance as a small applied field results in a large change in magnetization. Moreover, Brillouin light scattering experiments have shown interesting properties near the bending field, where the measured magnon frequency reaches a minimum value [4].

In this paper, we study the dynamics of a model of a hard-soft-hard exchange spring system in the vicinity of the bending field. We have chosen material parameters similar to a $\mathrm{DyFe}{ }_{2} / \mathrm{YFe}_{2} / \mathrm{DyFe}_{2}$ sample, which has been the subject of experimental investigation in our group.

\section{MODEL}

The system is a thin film made of one central magnetically soft $\mathrm{YFe}_{2}$ layer sandwiched between two thinner magnetically

Digital Object Identifier 10.1109/TMAG.2007.892596

Color versions of one or more of the figures in this paper are available online at http://ieeexplore.ieee.org.
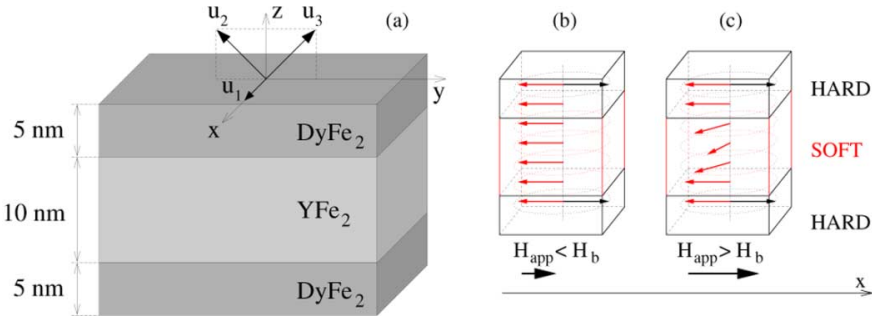

Fig. 1. (a) Geometry of the system and the orientation of the easy axes $u_{1}, u_{2}$, and $u_{3}$ for the cubic anisotropy. Its initial configuration (b) minimizes the energy when $H_{\text {app }}<H_{b}$, (c) but for higher fields an exchange spring is developed.

hard $\mathrm{DyFe}_{2}$ layers [see Fig. 1(a)]. Three different kinds of atoms are present in this system: one rare earth metal (Dy) and two transition metals (Fe and Y). The yttrium has negligible magnetic moment: only the iron moments contribute to the magnetization of the central layer. Conversely, in the two external layers both the atomic species of the $\mathrm{DyFe}_{2}$ compound give a relevant contribution to the magnetization. For the temperature considered here $(100 \mathrm{~K})$, the magnetic moment of iron is weaker than the one of dysprosium and is forced to be antiparallel to it by the strong antiferromagnetic coupling, which makes the $\mathrm{DyFe}_{2}$ a ferrimagnet. Moreover, Dy experiences a strong magnetocrystalline cubic anisotropy which makes the two external layers magnetically hard. The $\mathrm{Fe}-\mathrm{Fe}$ exchange coupling is even stronger than the Dy-Fe coupling [5] and opposes to the spatial variation in the orientation of the magnetizations. The coupling occurs also across the boundary surfaces between the layers. This is what allows the system to develop exchange spring configurations, keeping the iron moments bounded to the dysprosium moments of the hard layers at the boundaries. The effects of strain on the magnetic anisotropy of MBE-grown $\mathrm{DyFe}_{2} / \mathrm{YFe}_{2}$ superlattices are negligible at temperatures below $\sim 100 \mathrm{~K}[6]$.

We study the system with a unidimensional model along the out-of-plane direction, neglecting spatial variations of the magnetization across the plane of the layers. The configuration of the magnetic moments in the system is represented by two continuous magnetization fields $\mathbf{M}_{\mathrm{Dy}}(z)$ and $\mathbf{M}_{\mathrm{Fe}}(z)$ defined over the 1-D space: $\mathbf{M}_{\mathrm{Dy}}$ is the moment density of Dy atoms in $\mathrm{DyFe}_{2}$ 
and $\mathrm{M}_{\mathrm{Fe}}$ is the moment density of Fe atoms in $\mathrm{DyFe}_{2}$ and $\mathrm{YFe}_{2}$. Their norms are $M_{\mathrm{Dy}}=\left\|\mathbf{M}_{\mathrm{Dy}}\right\|$ and $M_{\mathrm{Fe}}=\left\|\mathbf{M}_{\mathrm{Fe}}\right\|$, which at the temperature of $100 \mathrm{~K}$ are $M_{\mathrm{Dy}}=1.73 \times 10^{6} \mathrm{~A} / \mathrm{m}$ and, for both $\mathrm{DyFe}_{2}$ and $\mathrm{YFe}_{2}, M_{\mathrm{Fe}}=0.55 \times 10^{6} \mathrm{~A} / \mathrm{m}$. The Brown's theory of micromagnetics is used to derive the effective fields acting on $\mathbf{M}_{\mathrm{Dy}}$ and $\mathbf{M}_{\mathrm{Fe}}$ :

$$
\begin{aligned}
\mathbf{H}_{\mathrm{Dy}} & =\mathbf{H}_{\mathrm{app}}+\mathbf{H}_{\mathrm{d}}+\mathbf{H}_{\mathrm{anis}}+\left(J / \mu_{0}\right) \mathbf{M}_{\mathrm{Fe}} \\
\mathbf{H}_{\mathrm{Fe}} & =\mathbf{H}_{\mathrm{app}}+\mathbf{H}_{\mathrm{d}}+\mathbf{H}_{\mathrm{exch}}+\left(J / \mu_{0}\right) \mathbf{M}_{\mathrm{Dy}}
\end{aligned}
$$

The applied field acts on both dysprosium and iron. $\mathbf{H}_{\mathrm{Dy}}$ also receives a cubic anisotropy contribution, whose three axes are shown in Fig. 1(a). The three coefficients used in the anisotropy expansion [7] are $K_{1}=33.9 \times 10^{6} \mathrm{~J} / \mathrm{m}^{3}, K_{2}=-16.2 \times 10^{6}$ $\mathrm{J} / \mathrm{m}^{3}$, and $K_{3}=16.4 \times 10^{6} \mathrm{~J} / \mathrm{m}^{3}$. The iron experiences an exchange field $\mathbf{H}_{\text {exch }}=\left(2 A / \mu_{0} M_{\mathrm{Fe}}\right) \nabla^{2} \mathbf{m}_{\mathrm{Fe}}$, where $A=1.46 \times$ $10^{-11} \mathrm{~J} / \mathrm{m}$ is the exchange coupling constant, $\mu_{0}$ is the free space permeability, and $\mathbf{m}_{\mathrm{Fe}}=\mathrm{M}_{\mathrm{Fe}} / M_{\mathrm{Fe}}$. The dipolar field $\mathbf{H}_{d}$ is calculated as $\mathbf{H}_{d}=-M_{z} \mathbf{z}$, where $M_{z}$ is the out-of-plane component of the total magnetization field and $\mathbf{z}$ is the unit vector which points towards the positive $z$ direction. This contribution evidently tries to reduce the surface magnetic charges, pulling the magnetization into the plane of the film. The Dy-Fe coupling is modeled by an extra energy term $U=J \int \mathbf{M}_{\mathrm{Dy}}$. $\mathbf{M}_{\mathrm{Fe}} d z$ in the Hamiltonian, where $J=2 \times 10^{-4} \mathrm{~N} / \mathrm{A}^{2}$ is the coupling constant [5] and the integral is done over the hard layers, where both magnetizations are defined. The Landau-Lifshitz-Gilbert equation is then used to calculate the dynamics of the magnetizations.

The unidimensional model is a severe approximation for a description of the switching processes, where domain walls can form and play an important role, but for our investigation near the bending field the model should be quite accurate.

\section{Results}

For the simulations, we use the Nmag micromagnetic simulation package [8], choosing the first-order finite element method (FEM) to discretize the space. The chosen mesh is a unidimensional lattice with constant spacing $\Delta z=0.5 \mathrm{~nm}$. We first calculate the hysteresis loop of the sample covering a wide range for the applied field $\mathbf{H}_{\mathrm{app}}$ : from $-60 \mathrm{~T}$ to $60 \mathrm{~T}$ in 250 steps (see Fig. 2). $\mathbf{H}_{\text {app }}$ is directed along the positive $x$-axis. To avoid the system to be trapped in an unstable equilibrium configuration, we follow the standard practice of adding a small constant deviation to the applied field: $\mathbf{H}_{\mathrm{dev}}=(0,1,1) \times 0.005 \mathrm{~T}$. The high field required for the switching (around $55 \mathrm{~T}$ ) represents a clear manifestation of Brown's paradox. In this paper, however, we are not concerned with this, since we are exploring a low-field region near the bending field, where the unidimensional model should be rather accurate. The bending field has been located around $H_{b} \approx 4.3 \mathrm{~T}$. The results, which are shown in Fig. 2, have been successfully validated against the ones obtained using the single-field model used by Zimmermann et al. [9].

After these preliminary calculations, we start the main simulation. We prepare the system in the configuration where $\mathbf{M}_{\mathrm{Dy}}$ lies in the plane of the film and points along the positive $x$-axis, while $\mathbf{M}_{\mathrm{Fe}}$ points along the opposite direction [see Fig. 1(b)]. This is an equilibrium configuration when the applied field

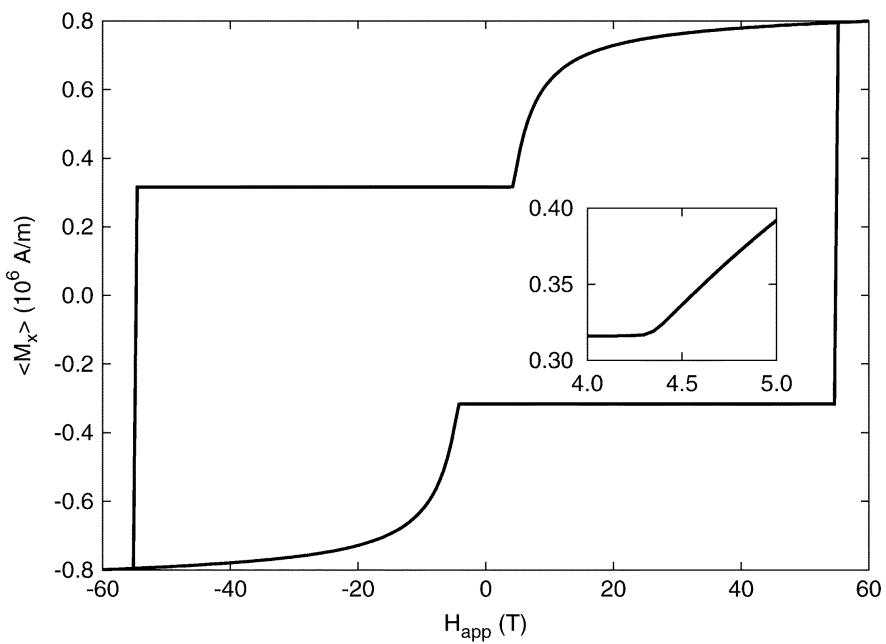

Fig. 2. Hysteresis loop. The field is applied in the in-plane $x$-direction. The curve around the bending field is shown in the inset in more detail.
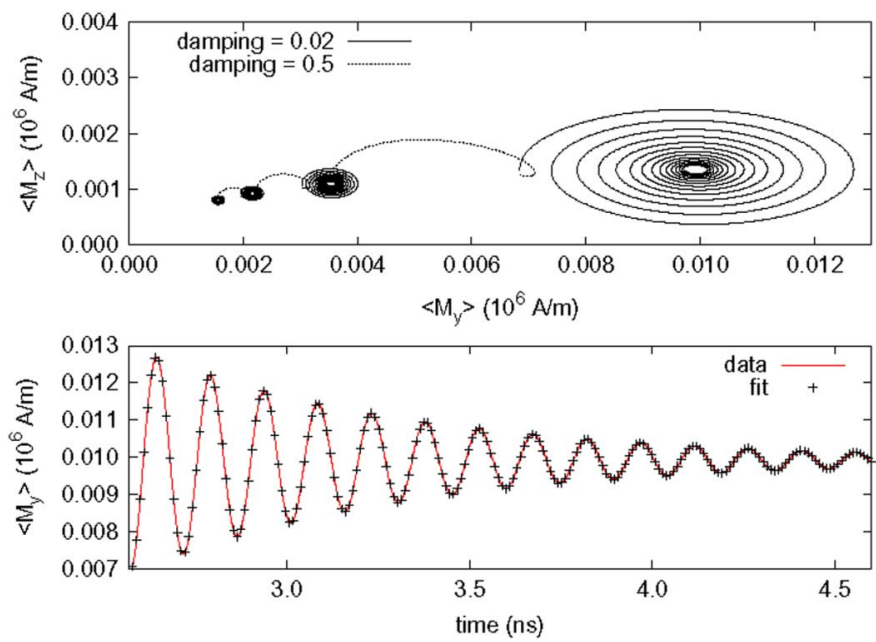

Fig. 3. Top: Trajectories of $\langle\mathbf{M}\rangle$ for $\mathbf{H}_{\text {app }}=3.60,3.80,4.00,4.20 \mathrm{~T}$. At each step, the system reaches the equilibrium configuration for $\mathbf{H}_{\text {app }}=\mathbf{H}_{n}$ with a high damping parameter $\alpha=0.5$ (dotted line). Then the field is changed to $\mathbf{H}_{\text {app }}=\mathbf{H}_{n}+\Delta \mathbf{H}$ and $\alpha=0.02$ is used to study the response (solid line) to the small increase in the field intensity. Bottom: $\left\langle M_{y}\right\rangle$ is also plotted as a function of time for $\mathbf{H}_{\mathrm{app}}=4.20 \mathrm{~T}$ together with the fitted curve.

$\mathbf{H}_{\text {app }}$ is zero, which is the situation we choose to start with in our simulation. To study a certain applied field $\mathbf{H}_{\text {app }}$, we first set the damping parameter in the Landau-Lifshitz-Gilbert equation to a high value $(\alpha=0.5)$, to quickly reach convergence. We then set the damping to the realistic value $(\alpha=0.02)$, increase the field intensity by a small amount $\Delta H$ and let the system evolve towards equilibrium. The trajectory of the mean magnetization $\langle\mathbf{M}\rangle$ is then studied carefully. This procedure is repeated in sequence for many values of $\mathbf{H}_{\mathrm{app}}$ near the bending field. In particular, we choose values of $\mathbf{H}_{\text {app }}$ which all point along the positive $x$-direction with intensities which range from 0 to $12 \mathrm{~T}$. We use more values of $\mathbf{H}_{\text {app }}$ near the bending field, to better resolve the behavior of the system in that region. For all the considered applied fields, we use the same value of $\Delta H=0.05 \mathrm{~T}$. A piece of the $\langle\mathbf{M}\rangle$ trajectory, projected in the $y-z$-plane, is shown in Fig. 3. These curves should reflect the changes in the magnetization of the soft layer only 

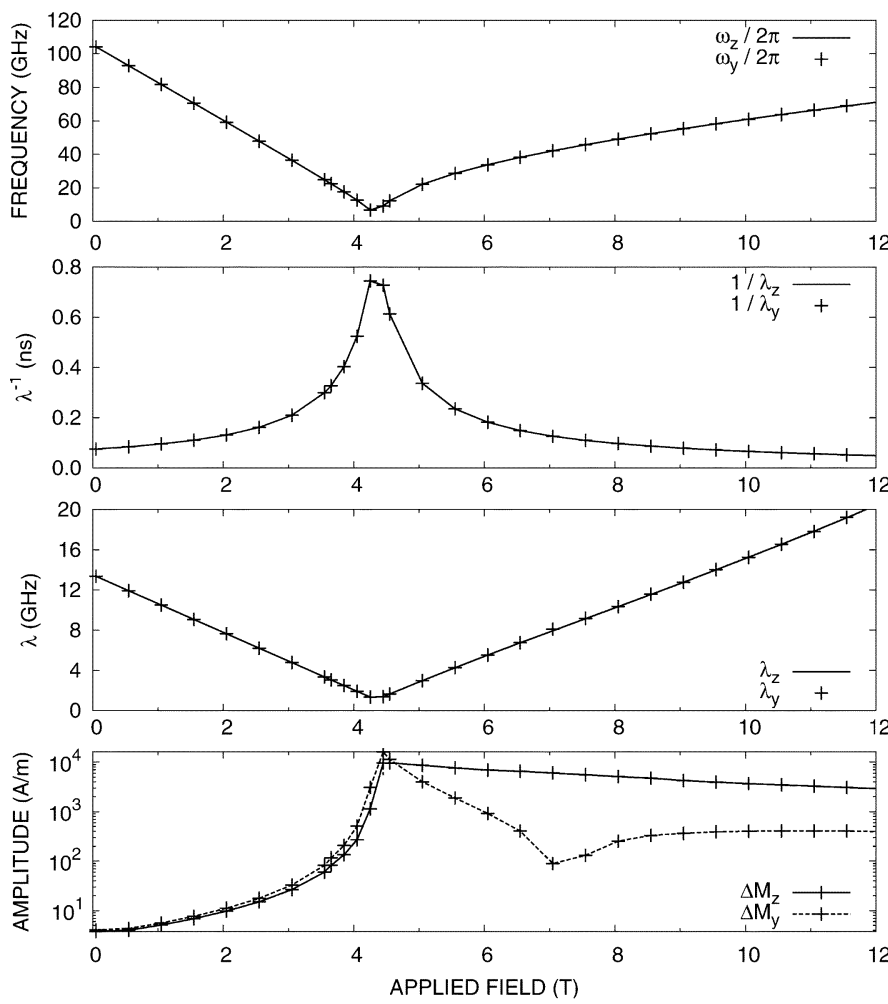

Fig. 4. Parameters obtained from the fits. In the top three graphs, solid lines are used for the values obtained fitting the $z$-component $\left\langle M_{z}\right\rangle$, while the crosses are used for $\left\langle M_{y}\right\rangle$.

because the magnetization of the hard layers is almost fixed for the considered range of the applied field. The alternating switching between the high and the low damping parameter can be distinguished clearly. The shape of these curves are all similar from one to the other: they are spirals compressed along the out-of-plane $z$-direction. The graph shows only a few trajectories for $H_{\text {app }}<H_{b}$. For higher fields the spirals become much wider. In Fig. 3 (bottom), the $y$-component of the mean magnetization is plotted as a function of time for the case $H_{\text {app }}=4.20 \mathrm{~T}$, just below the bending field. We fit these results to the equations of motion for a damped harmonic oscillator

$$
\begin{aligned}
& M_{y}(t)=M_{y, 0}+\Delta M_{y} e^{-\lambda_{y} t} \cos \left(\omega_{y}\left(t-t_{0}\right)+\varphi_{y}\right) \\
& M_{z}(t)=M_{z, 0}+\Delta M_{z} e^{-\lambda_{z} t} \cos \left(\omega_{z}\left(t-t_{0}\right)+\varphi_{z}\right) .
\end{aligned}
$$

We analyze the trajectories individually, to extract the frequencies, the decay times and the amplitudes of the oscillations produced as a reaction to the small perturbation $\Delta H$. Fig. 4 collects the results of the fits. The corresponding curves closely match the data. An example is shown in Fig. 3. The graphs in Fig. 4 clearly show that near the bending field the dynamical reaction of the system is amplified: the same perturbation $\Delta H$ produces wider oscillations with smaller frequency and which last longer (higher decay time $\lambda^{-1}$ ). The plot of the frequencies shows a minimum near $H_{b}$ and a qualitative behavior which is consistent with previous experimental and theoretical results [4]. The amplitudes, whose magnitude depends on the value chosen for $\Delta H$, show an interesting feature: the shapes of the spiral trajec- tories (Fig. 3) change with the applied field, being elongated in the in-plane $y$-direction for $H_{\text {app }} \ll H_{b}$ and in the out-of-plane the $z$-direction for $H_{\text {app }} \gg H_{b}$. We have repeated our studies increasing $\Delta H$ by a factor of 5 and decreasing it by a factor of 10. This does affect frequencies and decay times by less than $0.015 \%$ and $0.2 \%$, respectively.

\section{CONCLUSION}

We have carried out micromagnetic simulations of the dynamics of a tri-layer $\mathrm{DyFe}_{2} / \mathrm{YFe}_{2} / \mathrm{DyFe}_{2}$ exchange spring system with realistic crystal field anisotropy near the bending field, showing how the magnetization reacts to small increments in the intensity of the applied field. We have shown that the dynamics of the components of the mean magnetization can be fitted with excellent results against the equation of motion of damped harmonic oscillators. We obtained the frequencies, the decay times, and the amplitudes of these oscillations and found that they have a minimum frequency, a maximum decay time, and a maximum amplitude near the bending field.

\section{ACKNOWLEDGMENT}

This work was supported by the Engineering and Physical Sciences Research Council (EPSRC), U.K. (GR/T09156, GR/S95824).

\section{REFERENCES}

[1] D. Süß, T. Schrefl, R. Dittrich, M. Kirschner, F. Dorfbauer, G. Hrkac, and J. Fidler, "Exchange spring recording media for areal densities up to $10 \mathrm{Tbit} / \mathrm{in}^{2}$," IEEE Trans. Magn., vol. 290-291, pp. 551-554, Apr. 2005.

[2] R. Skomski and J. M. D. Coey, "Giant energy product in nanostructured two-phase magnets," Phys. Rev. B, vol. 48, no. 21, pp. 15812-15816, Dec. 1993.

[3] M. Sawicki, G. J. Bowden, P. A. J. de Groot, B. D. Rainford, J.-M. L. Beaujour, R. C. C. Ward, and M. R. Wells, "Exchange springs in antiferromagnetically coupled $\mathrm{DyFe}_{2} / \mathrm{YFe}_{2}$ superlattices," Phys. Rev. $B$, vol. 62 , no. 9 , pp. 5817-5820, Sep. 2000.

[4] M. Grimsditch, R. Camley, E. E. Fullerton, S. Jiang, S. D. Baderand, and C. H. Sowers, "Exchange-spring systems: Coupling of hard and soft ferromagnets as measured by magnetization and Brillouin light scattering," J. Appl. Phys., vol. 85, pp. 5901-5904, Apr. 1999.

[5] M. J. Bentall, R. A. Cowley, W. J. L. Buyers, Z. Tun, W. Lohstroh, R. C. C. Ward, and M. R. Wells, "Magnetic structures of laves phase superlattices," J. Phys.: Condens. Matter, vol. 15, pp. 4301-4330, Jul. 2003.

[6] A. Mougin, C. Dufour, K. Dumesnil, and P. Mangin, "Strain-induced magnetic anisotropy in single-crystal $r \mathrm{Fe}_{2}(110)$ thin films $\left(r=\mathrm{Dy}, \mathrm{Er}, \mathrm{Tb}, \mathrm{Dy}_{0.7} \mathrm{~Tb}_{0.3}, \mathrm{Sm}, \mathrm{Y}\right)$," Phys. Rev. $B$, vol. 62, no. 14 , pp. $9517-9531$, Oct. 2000

[7] K. N. Martin, P. A. J. de Groot, B. D. Rainford, K. Wang, G. J. Bowden, J. P. Zimmermann, and H. Fangohr, "Magnetic anisotropy in the cubic Laves $\mathrm{REFe}_{2}$ intermetallic compounds," J. Phys.: Condens. Matter, vol. 18, pp. 459-478, Jan. 2006.

[8] Univ. Southampton, Southampton, U.K., "Nmag-A micromagnetic simulation environment," (2007). [Online]. Available: http://www. soton.ac.uk/ fangohr/nsim/nmag

[9] J. P. Zimmermann, K. Martin, G. Bordignon, R. P. Boardman, T. Fischbacher, G. J. Bowden, A. A. Zhukov, H. Fangohr, and P. A. J. de Groot, "Micromagnetic simulation of the magnetic exchange spring system DyFe ${ }_{2} / \mathrm{DyFe}_{2}$," J. Appl. Phys., vol. 99, Apr. 2006, p. 08B904.

Manuscript received February 2, 2007 (e-mail: franchin@soton.ac.uk). 